\title{
CONTINUITY OF AREA FOR HARMONIC SURFACES WITH BOUNDARIES OF UNIFORMLY BOUNDED LENGTH
}

\author{
K. H. CARLSON AND L. C. YOUNG
}

The object of this note is to supplement a paper by Young [1],1 On the isoperimetric ratio for a harmonic surface, by answering in the affirmative a question raised by Morse and Tompkins [2]. Continuity of area as the harmonic surface $S^{*}$ shrinks to a point is dealt with by Young by means of his main inequality. Here the same machinery deals with the more general case in which $S^{*}$ approximates another surface $S$.

In what follows $r=|u+i v|, D$ is the disc $r \leqq 1, R$ its rim, and, in correspondence with a real number $t$ where $0<t<1, D^{-}$is the concentric disc $r \leqq 1-t$ and $R^{+}$is the annulus $1-t \leqq r \leqq 1$. We shall divide $R^{+}$into $n$ parts $\sigma$ by the $n$ segments $\lambda$ in which it is met by the lines amp $(u+i v)=2 \pi k / n$, where $-n / 2<k \leqq n / 2$. The suffixes $t$, or $k, n, t$ in $D^{-}$and $R^{+}$, or in $\sigma$ and $\lambda$, are understood to have been dropped.

$S$ and $S^{*}$ denote continuous vector functions $x(u, v)$ and $x^{*}(u, v)$ on $D$ and it is assumed that $x^{*}(u, v)$ is the harmonic extension to $D$ of a continuous vector function on $R$. We shall assume further that $(1-r)\left|x_{u}+i x_{v}\right|$ is bounded in $D$, and we denote by $b$ its supremum; $b^{*}$ is similarly defined in terms of $S^{*}$ [and is finite since $x^{*}(u, v)$ is harmonic].

We write $d\left(S, S^{*}\right)$ and $\rho\left(S, S^{*}\right)$ respectively for the suprema in $D$ of the expressions $\left|x(u, v)-x^{*}(u, v)\right|$ and $(1-r)\left|\left(x_{u}-x_{u}^{*}\right)+i\left(x_{v}-x_{v}^{*}\right)\right|$. Moreover, given any subset $E$ of $D$ whose boundary is a simple closed curve $C$, we write $\delta(E)$ for the diameter of the set of values $x(E)$ taken by $x(u, v)$ in $E$, and $A(E), L(E)$ respectively for the area and boundary-length defined by the expressions

$$
\iint_{E}\left\{x_{u}^{2} x_{v}^{2}-\left(x_{u} x_{v}\right)^{2}\right\}^{1 / 2} d u d v, \quad \int_{C}|d x| ;
$$

$\delta^{*}(E), A^{*}(E), L^{*}(E)$ denote the similar quantities for $S^{*}$ and the argument $E$ is omitted when $E=D$.

Lemma 1. For the sum of the angles $\alpha$ subtended at $\cos \gamma+i \sin \gamma$ by the $n$ segments $\lambda$, we have the estimate $\sum \alpha<\pi$ provided that $t \leqq \eta(n)$ where $\eta(n)=[n(1+\log n)]^{-1}$.

Received by the editors January 22, 1951.

1 Numbers in brackets refer to the references at the end of the paper. 
Proof. We first estimate the angle $\alpha$, subtended by the single segment $\lambda=A B$ on which amp $(u+i v)=\theta$, in terms of $\beta=|\theta-\gamma| / 2$ where $\theta-\gamma$ is reduced $\bmod 2 \pi$ so that $2 \beta \leqq \pi$. The diameter $A C$ containing $A B$ subtends a right angle at $M=\cos \gamma+i \sin \gamma$ and we have two expressions for the sine of the angle $\phi$ subtended by $M C$ at $B$ :

$2 \sin \beta \sin \alpha / t \equiv \overline{A M} \sin \alpha / \overline{A B}=\sin \phi$

$$
=\overline{M C} \cos \alpha / \overline{B C} \equiv 2 \cos \beta \cos \alpha /(2-t) .
$$

Hence if $\beta \neq 0$ we find that $\alpha<\tan \alpha=t \cot \beta /(2-t)<t \cot \beta<t / \beta$; moreover, in any case, $\alpha \leqq \pi / 2$.

To establish our lemma, in which we may suppose by circular symmetry that $0 \leqq \gamma \leqq \pi / n$, we have, on denoting by $\sum^{\prime}$ and $\Sigma^{\prime \prime}$ sums for $-n / 2<k<0$ and $0<k \leqq n / 2$,

$$
\begin{aligned}
\sum \alpha< & \frac{\pi}{2}+\left(\sum^{\prime}+\sum^{\prime \prime}\right) \frac{t}{\beta}=\frac{\pi}{2}+t \sum^{\prime}\left(\frac{\gamma}{2}-\frac{k \pi}{n}\right)^{-1} \\
& +t \sum^{\prime \prime}\left(\frac{k \pi}{n}-\frac{\gamma}{2}\right)^{-1} \\
& \leqq \frac{\pi}{2}+t \sum^{\prime}\left(-\frac{k \pi}{n}\right)^{-1}+t \sum^{\prime \prime}\left[\left(k-\frac{1}{2}\right) \frac{\pi}{n}\right]^{-1} \\
& \leqq \frac{\pi}{2}+\frac{2 n t}{\pi}\left\{1+\frac{1}{2}+\cdots+\frac{1}{n-1}\right\} \\
& <\frac{\pi}{2}+\frac{2 t}{\pi} n(1+\log n)<\frac{\pi}{2}[1+\operatorname{tn}(1+\log n)] \leqq \pi .
\end{aligned}
$$

LEMma 2. For every $S^{*}$ the inequality $t \leqq \eta(n)$ implies $\sum L^{*}(\sigma)$ $\leqq 5 L^{*}$.

Proof. Let $\Lambda=\sum \int_{\lambda}|d x *|$. By Young's variant [1, p. 400, (3.2)] of F. Carlson's inequality [3], we have, since $x^{*}$ is harmonic,

$$
\Lambda \leqq \pi^{-1} \int_{R}\left(\sum \alpha\right)\left|d x^{*}\right|<\int_{R}\left|d x^{*}\right|=L^{*},
$$

provided that $t \leqq \eta(n)$. Our assertion now follows from the relation $\sum L^{*}(\sigma)=2 \Lambda+L^{*}+L^{*}\left(D^{-}\right)$where the last term on the right cannot exceed $2 L^{*}$ (by $\left[1\right.$, p. 405 middle of page]; actually $L^{*}\left(D^{-}\right) \leqq L^{*}$ ).

LEмma 3. There is an absolute constant $K$ and, given $S$ and $\epsilon>0$, a number $t=t_{0}(\epsilon, S)<\epsilon$, such that the inequality $d\left(S, S^{*}\right)<\epsilon$ implies $A^{*}\left(R^{+}\right) \leqq \epsilon K L^{*}$.

Proof. By uniform continuity of $x(u, v)$, there exist $n=n(\epsilon, S)$ and $t=t_{0}(\epsilon, S) \leqq \eta(n)$, so that $\delta(\sigma)<\epsilon$. This evidently insures $\delta^{*}(\sigma)$ 
$<3 \epsilon$, while by Lemma $2, \sum L^{*}(\sigma) \leqq 5 L^{*}$. Consequently (applying Young's isoperimetric inequality $A \leqq K L d$ of [1] to the harmonic surface $x^{*}(u, v)$ on $\sigma$ and summing),

$$
A^{*}\left(R^{+}\right)=\sum A^{*}(\sigma) \leqq K \sum L^{*}(\sigma) \delta^{*}(\sigma) \leqq 15 \epsilon K L^{*},
$$

which is of the asserted form, with $15 K$ for $K$.

LEMMA 4. There is an absolute constant $K$ so that the inequality $\rho\left(S, S^{*}\right)<t^{4}$ implies $\left|A\left(D^{-}\right)-A^{*}\left(D^{-}\right)\right| \leqq K t\left(b+b^{*}\right)^{3 / 2}$.

Proof. Since the quantities $\left|x_{u}+i x_{v}\right| / b,\left|x_{u}^{*}+i x_{v}^{*}\right| / b^{*}$, and $\left|\left(x_{u}-x_{u}^{*}\right)+i\left(x_{v}-x_{v}^{*}\right)\right| / \rho\left(S, S^{*}\right)$ do not exceed $(1-r)^{-1}$, the expression $U=x_{u}^{2} x_{v}^{2}-\left(x_{u} x_{v}\right)^{2}$ differs $^{2}$ from the corresponding expression $U^{*}$ by at most $K^{2}(1-r)^{-4} \rho\left(S, S^{*}\right)\left(b+b^{*}\right)^{8}$ where $K$ is an absolute constant. Since

$$
\begin{aligned}
\left|U^{1 / 2}-U^{* 1 / 2}\right| & \leqq\left\{\left|U^{1 / 2}-U^{* 1 / 2}\right|\left(U^{1 / 2}+U^{* 1 / 2}\right)\right\}^{1 / 2} \\
& =\left|U-U^{*}\right|^{1 / 2}
\end{aligned}
$$

it follows that

$$
\begin{aligned}
\left|A\left(D^{-}\right)-A^{*}\left(D^{-}\right)\right| & \leqq \iint_{D^{-}}\left|U-U^{*}\right|^{1 / 2} d u d v \\
& =2 \pi K t^{2}\left(b+b^{*}\right)^{3 / 2} \int_{0}^{1-t}(1-r)^{-2} r d r
\end{aligned}
$$

which implies our assertion, because

$$
\int(1-r)^{-2} r d r \leqq \int(1-r)^{-2} d r=t^{-1}-1<t^{-1} .
$$

LEMMA 5. Given $S$ and the constant $N$ and given $\epsilon^{\prime}>0$, there exists $\epsilon^{\prime \prime}=\epsilon^{\prime \prime}\left(S, N, \epsilon^{\prime}\right)>0$ such that the relations $\rho\left(S, S^{*}\right)+d\left(S, S^{*}\right)<\epsilon^{\prime \prime}$, $L^{*} \leqq N, A<\infty$ together imply $\left|A-A^{*}\right|<\epsilon^{\prime}$ for every harmonic $S^{*}$.

Proof. Clearly $\delta^{*} \leqq L^{*}$ and by an inequality of Schwarz $[1$, p. 398, (2.3)] $b^{*} \leqq 2 \delta^{*}$. Moreover $b \leqq b^{*}+\rho\left(S, S^{*}\right)$, so that $b<\infty$. We may therefore suppose $N \geqq b$ without loss of generality (since $b$ depends only on $S$ ) and we then have $b+b^{*} \leqq 3 N$.

Given $\epsilon>0$, we now determine $t=t(\epsilon, S)<t_{0}(\epsilon, S)$, where $t_{0}(\epsilon, S)$ is defined as in Lemma 3, so that

$$
A\left(R^{+}\right)<\epsilon .
$$

\footnotetext{
$2\left|x_{u}^{2} x_{v}^{2}-x^{*}{ }_{u} x_{v}^{* 2}\right|=\left|x_{v}^{2}\left(x_{u}+x_{u}^{*}\right)\left(x_{u}-x_{u}^{*}\right)+x_{u}^{*}\left(x_{v}+x_{v}^{*}\right)\left(x_{v}-x_{v}^{*}\right)\right| \leqq 2\left[\left(b+b^{*}\right) /(1-r)\right]^{3}$ $\cdot \rho\left(S, S^{*}\right) /(1-r)$. Similarly this expression majorizes $\left|\left(x_{u}^{*} x_{v}^{*}\right)^{2}-\left(x_{u} x_{v}\right)^{2}\right|$.
} 
This is possible since $A$ is finite. Then the inequality $\rho\left(S, S^{*}\right)$ $+d\left(S, S^{*}\right)<t^{4}$ implies on one hand by Lemma 3 (since $t^{4}<t<\epsilon$ )

$$
A^{*}\left(R^{+}\right) \leqq K N \epsilon \text {, }
$$

and on the other hand by Lemma 4

$$
\left|A\left(D^{-}\right)-A^{*}\left(D^{-}\right)\right|<K \epsilon(3 N)^{3 / 2}
$$

Hence by addition the quantity

$$
\left|A-A^{*}\right|=\left|A\left(R^{+}\right)-A^{*}\left(R^{+}\right)+\left\{A\left(D^{-}\right)-A^{*}\left(D^{-}\right)\right\}\right|
$$

is less than $\epsilon+K N \epsilon+K \epsilon(3 N)^{3 / 2}=\epsilon^{\prime}$ provided our hypotheses are satisfied and $\epsilon^{\prime \prime}=t^{4}$ where $t=t(\epsilon, S)$. This completes the proof.

Continuity Theorem. If $S$ is harmonic and $N$ fixed, then for harmonic $S^{*}$ the relations $d\left(S, S^{*}\right) \rightarrow 0$ and $L^{*} \leqq N$ imply $A^{*} \rightarrow A$.

Proof. By semi-continuity of area we may suppose $A<\infty$. It is now sufficient to apply Lemma 5 with $3 \epsilon^{\prime \prime}$ in place of $\epsilon^{\prime \prime}$ since $\rho\left(S, S^{*}\right) \leqq 2 d\left(S, S^{*}\right)$ by the inequality of Schwarz already referred to.

The theorem just proved answers the question raised by Morse and Tompkins [2, p. 828]. We do not know, however, whether the result remains true if $d\left(S, S^{*}\right)$ is replaced by the Fréchet distance of the harmonic surfaces $S$ and $S^{*}$.

\section{REFERENCES}

1. L. C. Young, Proc. London Math. Soc. (2) vol. 49 (1947) pp. 396-408.

2. Marston Morse and C. Tompkins, Amer. J. Math. vol. 63 (1941) pp. 825-838.

3. F. Carlson, Arkiv för Matematik, Astronomi och Fysik vol. 29B (1943) no. 11.

UNIVERSITY OF WISCONSIN 